\title{
OLTC Transformer Model Connecting 3-Wire MV with 4-Wire Multigrounded LV Networks
}

Evangelos E. Pompodakis a, Georgios C. Kryonidis ${ }^{\text {a }}$, Minas C. Alexiadis ${ }^{\text {a }}$

${ }^{a}$ Power Systems Laboratory, School of Electrical and Computer Engineering, Aristotle University of Thessaloniki, Thessaloniki GR 54124, Greece

\begin{abstract}
This short communication presents a comprehensive model of on-load tap-changer (OLTC) transformers that connect 3wire medium voltage (MV) with 4-wire multigrounded low voltage (LV) networks. The proposed model enables the inclusion of the 3-wire MV network and the 4-wire multigrounded LV network into a single $Y_{\text {Bus }}$ matrix without any assumption or simplification. Its distinct feature is that the tap changer of the transformer is simulated outside the $\mathbf{Y}_{\text {Bus }}$ matrix, thus a refactorization of the $\mathbf{Y}_{\text {BUS }}$ matrix is not required in every tap change. The proposed transformer model has been validated in a 4Bus network, while its performance has been tested in the IEEE 8500-Node and IEEE 906-Bus test networks.
\end{abstract}

Keywords: Implicit $\mathrm{Z}_{\mathrm{BUS}}$ power flow, Multi-grounded networks, OLTC Transformer.

\section{Introduction}

Technological advances over the last years have made the OLTC MV/LV transformers a viable solution in distribution networks [1]. OLTC transformers allow LV network operators to 1) integrate economically renewable energy sources, 2) optimize grid topologies by reducing secondary substations, 3) stabilize industrial processes in volatile grids, and 4) complying economically with grid codes [1].

OLTC transformers have been studied in power flow literature assuming single-phase balanced [2] or 3-wire unbalanced configurations [3]. However, the accurate simulation of OLTC MV/LV transformers necessitates a precise transformer model that can be effectively combined with existing power flow algorithms, considering the distinct configurations that the MV and LV networks have. Although some transformer models have been proposed in power flow literature for integrating 3-wire MV and 4-wire multigrounded LV networks, they ignore the effect of tap changer [4].

Ideally, the tap changer should be included in the power flow with the tap variations not affecting the structure of the $\boldsymbol{Y}_{\boldsymbol{B} U \boldsymbol{S}}$ matrix. In this way, the factorization or inversion of the

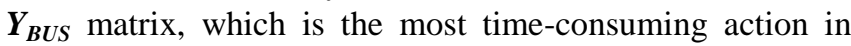
power flow is executed only once and not at every tap change. Thus, several power flow applications that require sequential tap variations e.g. voltage stability analysis, optimal power flow (OPF), Volt/Var control (VVC), optimal feeder reconfiguration (OFR) [2], [3], heuristic optimization are significantly accelerated.

This paper proposes an OLTC transformer model with the following distinct features:

- It has a Dyn configuration, which is by far the most common type of transformer supplying LV feeders. The model represents realistically the 3-wire nature of MV and the 4-wire multi-grounded nature of LV networks.

- The tap changer is considered in the form of current sources and it is not included in the $\boldsymbol{Y}_{\boldsymbol{B} \boldsymbol{U} \boldsymbol{S}}$ matrix of the network. Thus, any variation of the tap does not affect the structure of the $\boldsymbol{Y}_{\boldsymbol{B} U \boldsymbol{S}}$ matrix. This property is very important

«This research is co-financed by Greece and the European Union (European Social Fund- ESF) through the Operational Programme «Human Resources Development, Education and Lifelong Learning» in the context of the project "Strengthening Human Resources Research Potential via Doctorate Research" (MIS-5000432), implemented by the State Scholarships Foundation (IKY)». in $\mathrm{Z}_{\mathrm{BUS}}$-based power flow methods since the factorization and inversion of the $\boldsymbol{Y}_{\boldsymbol{B} U \boldsymbol{S}}$ is realized only once and not in every tap change, reducing in this way dramatically the computation time of the algorithm.

This short communication is an extension of [5], in which the authors implemented implicit $\mathrm{Z}_{\mathrm{BUS}}$ method to solve the power flow in 4-wire multigrounded LV networks operated in either grid-connected or islanded mode. By applying the proposed OLTC transformer model, the 3-wire upstream MV network can be also considered into the power flow. Thus, the estimation of several issues related to the interaction between the MV and LV networks e.g. overvoltages, unbalances and tap regulation is enabled.

\section{Network description}

A typical representation of a distribution network is shown in Fig. 1. It consists of a three wire MV network, which supplies a 4-wire multigrounded LV network through a Dyn11 transformer. More specifically, node 1 is the slack bus of the network with a constant reference voltage. The tap-changing MV windings of the transformer are connected to bus 2 in delta configuration. The neutral point of the LV side of the transformer is grounded though the impedance $\mathrm{Z}_{\mathrm{gr} 2}$, which represents the grounding impedance at the MV/LV substation. The LV nodes 3 and 4 are grounded with the impedances $Z_{\mathrm{gr} 3}$ and $\mathrm{Z}_{\mathrm{gr} 4}$, respectively, which represent the grounding impedances at the customer side.

The 3-wire MV line can be represented through a 3x3 admittance matrix consisting of the self- and mutualadmittances between the phases as follows:

$$
Y_{12}=\left[\begin{array}{lll}
y_{12}^{a a} & y_{12}^{a b} & y_{12}^{a c} \\
y_{12}^{b a} & y_{12}^{b b} & y_{12}^{b c} \\
y_{12}^{c a} & y_{12}^{c b} & y_{12}^{c c}
\end{array}\right]
$$

Similarly, the 4-wire multigrounded line can be represented through a $5 \times 5$ admittance matrix consisting of the self- and mutual-admittances between the phases, neutral and ground. For instance, the line between the nodes 3-4 is represented as follows:

$$
Y_{34}=\left[\begin{array}{lllll}
y_{34}^{a a} & y_{34}^{a b} & y_{34}^{a c} & y_{34}^{a n} & y_{34}^{a g} \\
y_{34}^{b a} & y_{34}^{b b} & y_{34}^{b c} & y_{34}^{b n} & y_{34}^{b g} \\
y_{34}^{c a} & y_{34}^{c b} & y_{34}^{c c} & y_{34}^{c n} & y_{34}^{c g} \\
y_{34}^{n a} & y_{34}^{n b} & y_{34}^{n c} & y_{34}^{n n} & y_{34}^{n g} \\
y_{34}^{g a} & y_{34}^{g b} & y_{34}^{g c} & y_{34}^{g n} & y_{34}^{g g}
\end{array}\right]
$$


Finally, the admittance matrix between the buses 2 '-3 is given in (3):

$$
\Upsilon_{2^{\prime} 3}=\left[\begin{array}{ccccc}
Z_{2^{\prime} 3 \alpha} & 0 & 0 & 0 & 0 \\
0 & Z_{2^{\prime} 3 b} & 0 & 0 & 0 \\
0 & 0 & Z_{2^{\prime} 3 c} & 0 & 0 \\
0 & 0 & 0 & Z_{2^{\prime} 3 n} & 0 \\
0 & 0 & 0 & 0 & Z_{g r 2^{\prime}}+Z_{2^{\prime} 3 g}
\end{array}\right]^{-1}
$$

where the impedances $Z_{2^{\prime} 3 \mathrm{a}}, Z_{2^{\prime} 3 \mathrm{~b}}$, and $Z_{2^{\prime} 3 \mathrm{c}}$ express the leakage impedances of the transformer. It is noted that the leakage impedances are represented at the LV side of the transformer, thus they are constant regardless the tap position [6]. $\mathrm{Z}_{2^{\prime} 3 \mathrm{n}}$ and $\mathrm{Z}_{2^{\prime} 3 \mathrm{~g}}$ denote the impedances of the neutral and ground between the buses 2'-3 and they tend to zero, while $\mathrm{Z}_{\mathrm{gr} 2^{\prime}}$ has been merged with $\mathrm{Z}_{2^{\prime} 3 \mathrm{~g}}$.

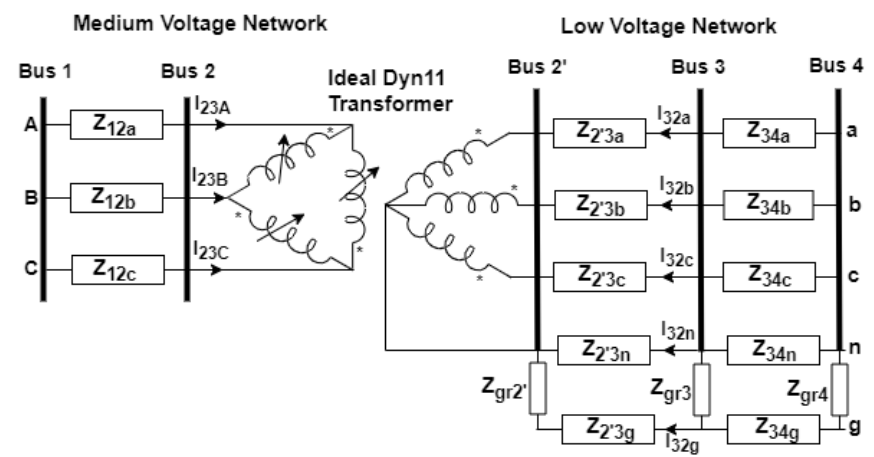

Fig. 1. Equivalent circuit of 4-Bus Network, consisting of a 3-wire MV and a 4-wire multigrounded LV network connected through a Dyn11 transformer.

\section{OLTC transformer model}

The turn ratio between primary and secondary windings for phase $i=\{a, b, c\}$ is defined as $a_{i}=\frac{N_{i}}{n_{i}}$, where $N_{i}$ and $n_{i}$ are the number of turns of primary and secondary windings, respectively. Assuming the tap changer is located at the primary side, which is usually the case for $\mathrm{MV} / \mathrm{LV}$ transformers, the turn ratios of the three phases are given in (4):

$$
\left[\begin{array}{l}
a_{a} \\
a_{b} \\
a_{c}
\end{array}\right]=\left[\begin{array}{l}
\left(1+0.00625 \cdot \operatorname{Tap}_{a}\right) \cdot a_{\text {nom }} \\
\left(1+0.00625 \cdot \operatorname{Tap}_{b}\right) \cdot a_{\text {nom }} \\
\left(1+0.00625 \cdot \operatorname{Tap}_{c}\right) \cdot a_{\text {nom }}
\end{array}\right]
$$

where $a_{\text {nom }}$ is the nominal turn ratio, while $\operatorname{Tap}_{i}$ of phase $i$ is a discrete variable that usually takes 33 values with a range of $\operatorname{Tap}_{i}=\{-16, \ldots, 0, \ldots, 16\}$.

Based on Fig. 1 and considering the star point of the transformer as reference point of the LV network, the voltage of fictitious bus $2^{\prime}$ is expressed as a function of the voltage of bus 2, as follows:

$$
\left[\begin{array}{l}
V_{2^{\prime}} a \\
V_{2^{\prime} b} \\
V_{2^{\prime}} c \\
V_{2^{\prime} n} \\
V_{2^{\prime}}
\end{array}\right]=\boldsymbol{T} \cdot\left[\begin{array}{l}
V_{2 A} \\
V_{2 B} \\
V_{2 C}
\end{array}\right] \quad \text { where } \quad \boldsymbol{T}=\left[\begin{array}{ccc}
\frac{1}{a_{a}} & \frac{-1}{a_{a}} & 0 \\
0 & \frac{1}{a_{b}} & \frac{-1}{a_{b}} \\
\frac{-1}{a_{c}} & 0 & \frac{1}{a_{c}} \\
0 & 0 & 0 \\
0 & 0 & 0
\end{array}\right]
$$

The line currents at the primary side of the transformer are given by (6).

$$
\boldsymbol{I}_{23}=\boldsymbol{A} \cdot \boldsymbol{Y}_{2^{\prime} \mathbf{3}}^{\text {mod }} \cdot\left[\boldsymbol{T} \cdot\left[\begin{array}{l}
V_{2 A} \\
V_{2 B} \\
V_{2 C}
\end{array}\right]-\left[\begin{array}{l}
V_{3 a} \\
V_{3 b} \\
V_{3 c} \\
V_{3 n} \\
V_{3 g}
\end{array}\right]\right]
$$

where $\boldsymbol{A}=\left[\begin{array}{ccc}\frac{1}{a_{a}} & 0 & \frac{-1}{a_{c}} \\ \frac{-1}{a_{a}} & \frac{1}{a_{b}} & 0 \\ 0 & \frac{-1}{a_{b}} & \frac{1}{a_{c}}\end{array}\right], \quad \boldsymbol{Y}_{2^{\prime} 3}^{\text {mod }}=\left[\begin{array}{ccccc}Z_{2^{\prime} 3 a}^{-1} & 0 & 0 & 0 & 0 \\ 0 & Z_{2^{\prime} 3 b}^{-1} & 0 & 0 & 0 \\ 0 & 0 & Z_{2^{\prime} 3 c}^{-1} & 0 & 0\end{array}\right]$

The line currents at the secondary side of the transformer are given by (7).

$$
\boldsymbol{I}_{\mathbf{3 2}}=\boldsymbol{Y}_{\mathbf{2}^{\prime} \mathbf{3}} \cdot\left[\begin{array}{l}
V_{3 a} \\
V_{3 b} \\
V_{3 c} \\
V_{3 n} \\
V_{3 g}
\end{array}\right]-\boldsymbol{T} \cdot\left[\begin{array}{l}
V_{2 A} \\
V_{2 B} \\
V_{2 C}
\end{array}\right]
$$

Combining (6) and (7), equation (8) is derived indicating that the transformer can be modeled by a set of self- and mutual-admittances as a function of the voltages and line currents of nodes 2 and 3. The square matrix in (8) includes the grounding impedance at the substation $\left(Z_{g r 2^{\prime}}\right)$ as well as the winding ratio $\left(a_{i}\right)$ and the leakage impedances $\left(Z_{2^{\prime} 3 a}, Z_{2^{\prime} 3 b}, Z_{2^{\prime} 3 c}\right)$ of the transformer.

$$
\left[\begin{array}{l}
\boldsymbol{I}_{\mathbf{2 3}} \\
\boldsymbol{I}_{\mathbf{3 2}}
\end{array}\right]=\left[\begin{array}{cc}
\boldsymbol{A} \cdot \boldsymbol{Y}_{\mathbf{2}^{\prime} \mathbf{3}}^{\boldsymbol{m o d}} \cdot \boldsymbol{T} & -\boldsymbol{A} \cdot \boldsymbol{Y}_{\mathbf{2}^{\prime} \mathbf{3}}^{\mathbf{m o d}} \\
-\boldsymbol{Y}_{\mathbf{2}^{\prime} \mathbf{3}} \cdot \boldsymbol{T} & \boldsymbol{Y}_{\mathbf{2}^{\prime} \mathbf{3}}
\end{array}\right] \cdot\left[\begin{array}{l}
V_{2 A} \\
V_{2 B} \\
V_{2 C} \\
V_{3 a} \\
V_{3 b} \\
V_{3 c} \\
V_{3 n} \\
V_{3 g}
\end{array}\right]
$$

Assuming that $\boldsymbol{A}_{\text {nom }}, \boldsymbol{T}_{\text {nom }}$ are the $\boldsymbol{A}$ and $\boldsymbol{T}$ matrices at the nominal turn ratio (namely $a_{\iota}=a_{\text {nom }}$ ), equation (8) can be expressed as shown in (9):

$$
\left[\begin{array}{l}
I_{23} \\
I_{32}
\end{array}\right]=\left[\begin{array}{cc}
A_{n o m} \cdot Y_{2^{\prime} 3}^{m o d} \cdot T_{n o m} & -A_{n o m} \cdot Y_{2^{\prime} 3}^{m o d} \\
-Y_{2^{\prime} 3} \cdot T_{n o m} & Y_{2^{\prime} 3}
\end{array}\right] \cdot\left[\begin{array}{l}
V_{2} \\
V_{3}
\end{array}\right]+\left[\begin{array}{l}
d I_{2} \\
d I_{3}
\end{array}\right]
$$

where

$\left[\begin{array}{l}d I_{2} \\ d I_{3}\end{array}\right]=\left[\begin{array}{cc}\left(A \cdot Y_{2^{\prime} 3}^{m o d} \cdot T-A_{n o m} \cdot Y_{2^{\prime} 3}^{m o d} \cdot T_{\text {nom }}\right) & \left(-A \cdot Y_{2^{\prime} 3}^{\text {mod }}+A_{\text {nom }} \cdot Y_{2^{\prime} 3}^{\text {mod }}\right) \\ \left(-Y_{2^{\prime} 3} \cdot T+Y_{2^{\prime} 3} \cdot T_{\text {nom }}\right) & 0\end{array}\right] \cdot\left[\begin{array}{c}V_{2} \\ V_{3}\end{array}\right]$

The square matrix in (9) is constant and can be included in the $\boldsymbol{Y}_{\boldsymbol{B} \boldsymbol{U} \boldsymbol{S}}$ matrix without requiring re-factorization at every tap change. The elements $\boldsymbol{d I}_{\mathbf{2}}, \boldsymbol{d I}_{\mathbf{3}}$ include the tap variables of the transformer and they are modeled as fictitious current sources outside the admittance matrix.

Figure 2 depicts the equivalent circuit of a Dyn11 transformer connecting a 3-wire MV with a 4-wire multigrounded LV network. The current sources are calculated by (11), while $\boldsymbol{Y}_{\boldsymbol{p} p}, \boldsymbol{Y}_{\boldsymbol{p} \boldsymbol{s}}, \boldsymbol{Y}_{\boldsymbol{s p}}, \boldsymbol{Y}_{\boldsymbol{s}}$ are expressed in (12). The equivalent circuit of Fig. 2a represents that part of the network shown in Fig. 2b. It is clarified, that although the existing analysis refers to a Dyn11 configuration, it can be also applied in all Dyn configurations by simply adapting the matrices $\boldsymbol{A}, \boldsymbol{T}, \boldsymbol{A}_{\text {nom }}, \boldsymbol{T}_{\text {nom }}$.

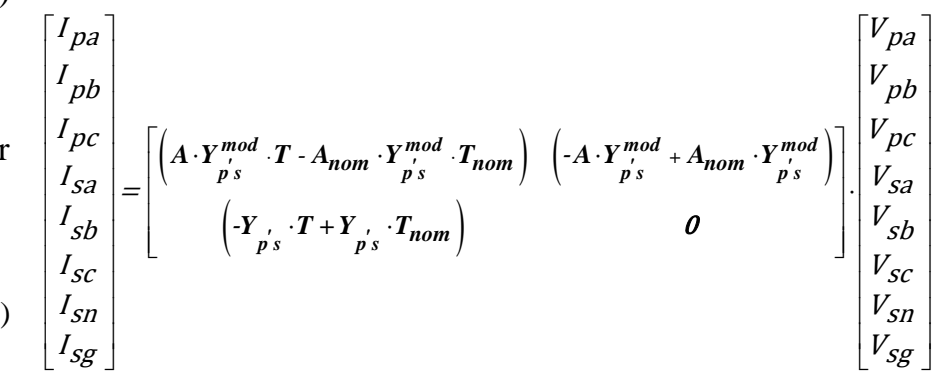




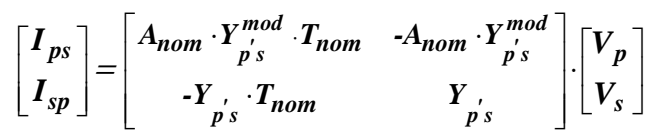

\section{Power flow of networks with OLTC}

In this section, the implementation of the proposed model into the power flow using the network of Fig. 1 is explained. The network of Fig. 1 is mathematically described by (13), as follows:

$$
\left[\begin{array}{c}
I_{1} \\
I_{2} \\
I_{3} \\
I_{4}
\end{array}\right]=\left[\begin{array}{cccc}
-Y_{12} & Y_{12} & 0 & 0 \\
Y_{12} & -Y_{12}-A_{n o m} \cdot Y_{2^{\prime} 3}^{m o d} \cdot T_{n o m} & A_{n o m} \cdot Y_{2^{\prime} 3}^{m o d} & 0 \\
0 & Y_{2^{\prime} 3} \cdot T_{n o m} & -Y_{2^{\prime} 3}-Y_{34} & Y_{34} \\
0 & 0 & Y_{34} & -Y_{34}
\end{array}\right] \cdot\left[\begin{array}{c}
V_{1} \\
V_{2} \\
V_{3} \\
V_{4}
\end{array}\right]
$$

The square matrix of (13) includes the 3-wire MV line between the node 1-2 described by (1), the 4-wire multigrounded line between the nodes 3-4 represented by (2) and the proposed OLTC model described in (12). The voltage and current vectors of (13) for each node $j=\{1,2,3$, 4 \} are expressed by the equations below:

$$
\begin{aligned}
& \boldsymbol{V}_{\mathbf{1}}=\left[\begin{array}{lll}
V_{1 a}, & V_{1 b}, & V_{1 c}
\end{array}\right]^{T}, \\
& \boldsymbol{V}_{\mathbf{2}}=\left[\begin{array}{lll}
V_{2 a}, & V_{2 b}, & V_{2 c}
\end{array}\right]^{T} \text {, }
\end{aligned}
$$

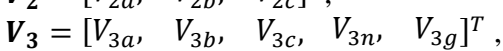

$$
\begin{aligned}
& \boldsymbol{V}_{\mathbf{4}}=\left[\begin{array}{lllll}
V_{4 a}, & V_{4 b}, & V_{4 c}, & V_{4 n}, & V_{4 g}
\end{array}\right]^{T} \text {. } \\
& \boldsymbol{I}_{\mathbf{1}}=\left[\begin{array}{lll}
I_{L 1 a}, & I_{L 1 b}, & I_{L 1 c}
\end{array}\right]^{T}, \\
& \boldsymbol{I}_{2}=\left[\begin{array}{lll}
I_{L 2 a}, & I_{L 2 b}, & I_{L 2 c}
\end{array}\right]^{T}+\left[\begin{array}{lll}
I_{p a}, & I_{p b}, & I_{p c}
\end{array}\right]^{T},
\end{aligned}
$$

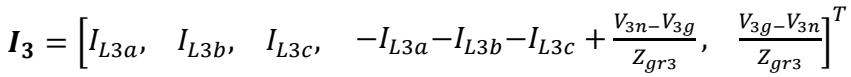

$$
\begin{aligned}
& +\left[\begin{array}{lllll}
I_{s a}, & I_{s b}, & I_{s c}, & I_{s n}, & I_{s g}
\end{array}\right]^{T}
\end{aligned}
$$

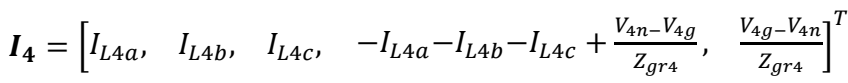

In the equations above, the voltage vectors $\boldsymbol{V}_{\boldsymbol{j}}$ include the voltage elements $V_{j y}$, which denote the voltage (in complex form) of node $j$ at conductor $y=\{a, b, c, n, g\}$. The current vectors $\boldsymbol{I}_{\boldsymbol{j}}$ include the load currents $I_{L j r}$ of node $j$ at phase $r=\{a, b, c\}$ as well as the currents flowing through the grounding resistances $Z_{\text {grx }}$ for $x=\{3,4\}$. The current vectors $\boldsymbol{I}_{2}$ and $\boldsymbol{I}_{\mathbf{3}}$ include also the compensating currents $\left(I_{p a}, I_{p b}, \ldots, I_{s n}, I_{s g}\right)$ of the proposed OLTC model, as shown in Fig. 2a and expressed in (11).

Assuming that the bus 1 is the slack bus, the first three rows of (13) are removed and (14) is obtained, as follows:

$$
\left[\begin{array}{c}
I_{2} \\
I_{3} \\
I_{4}
\end{array}\right]=\left[\begin{array}{cccc}
Y_{12} & -Y_{12}-A_{n o m} \cdot Y_{2^{\prime} 3}^{m o d} \cdot T_{n o m} & A_{n o m} \cdot Y_{2^{\prime} 3}^{m o d} & 0 \\
0 & Y_{2^{\prime} 3} \cdot T_{n o m} & -Y_{23}-Y_{34} & Y_{34} \\
0 & 0 & Y_{34} & -Y_{34}
\end{array}\right] \cdot\left[\begin{array}{l}
V_{1} \\
V_{2} \\
V_{3} \\
V_{4}
\end{array}\right]
$$

As a next step, the voltage variables $V_{3 n}, V_{3 g}, V_{4 n} V_{4 g}$ included in the current vectors $\boldsymbol{I}_{3}, \boldsymbol{I}_{\mathbf{4}}$ are transferred to the right-hand side of (14) and (15) is derived

$$
I_{\text {new }}=Y_{\text {new }} \cdot V
$$

where $\boldsymbol{I}_{\text {new }}$ and $\boldsymbol{Y}_{\text {new }}$ are the modified current and admittance matrices.

Finally, we define the final matrices $\boldsymbol{Y}_{\text {fin }}^{\prime}$ and $\boldsymbol{Y}_{\boldsymbol{f i n}}$. The first one consists of the first three columns of $\boldsymbol{Y}_{\text {new }}$, while the second one consists of the remaining columns so that $\boldsymbol{Y}_{\text {new }}=\left[\begin{array}{ll}\boldsymbol{Y}_{\text {fin }}^{\prime} & \boldsymbol{Y}_{\boldsymbol{f i n}}\end{array}\right]$. Equation (16) is then derived from Equation (15) by subtracting the product $\boldsymbol{Y}_{\text {fin }}^{\prime} \cdot \boldsymbol{V}_{\mathbf{1}}$ from both equation sides.

$$
-Y_{f i n}^{\prime} \cdot V_{1}+I_{\text {new }}=-Y_{f i n}^{\prime} \cdot V_{1}+Y_{n e w} \cdot V
$$

Using (16), we finally derive (17), which is iteratively solved until a certain preset tolerance is reached. In (17), $k$ denotes the iteration number, while the vector $\boldsymbol{V}_{\text {fin }}$ contains the voltages of all nodes except the slack node $\left(\boldsymbol{V}_{\mathbf{1}}\right)$.

$$
\boldsymbol{Y}_{\boldsymbol{f i n}}^{-1} \cdot\left[-\boldsymbol{Y}_{\boldsymbol{f i n}}^{\prime} \cdot \boldsymbol{V}_{\mathbf{1}}+\boldsymbol{I}_{\text {new }}\right]^{k}=\boldsymbol{V}_{\boldsymbol{f i n}}{ }^{k+1}
$$

In equation (17), the matrix $\boldsymbol{Y}_{\boldsymbol{f i n}}$ consists of constant elements regardless the tap positions, thus it is factorized only once. The tap variations of OLTC are represented in the current vector $\boldsymbol{I}_{\text {new }}$ through the compensating currents of (11). It is pointed out that although the analysis above concerns a small 4-bus non-realistic network, the same analysis can be applied to solve the power flow of large networks. More details about the $\mathrm{Z}_{\mathrm{BUS}}$ power flow solver referred above can be found in [5], [8].

\section{Validation of the OLTC transformer model}

The proposed OLTC transformer model is validated against Simulink in the 4-bus network of Fig. 1. The network is based on the IEEE 4-bus test feeder, but it was slightly modified to represent a 3-wire MV and a 4-wire multigrounded LV network. The transformer has a Dyn11 configuration. All the parameters are shown in Table 1.

Table 2 depicts indicatively the phase-to-phase and phaseto-neutral voltage magnitudes of buses 2 and 4, as they were calculated with Simulink and the proposed approach. As shown, the results of the proposed approach are in full agreement with those of Simulink confirming the accuracy of the proposed OLTC transformer model.

Table 1

Parameters of the 4-Bus network.

\begin{tabular}{|c|c|}
\hline Line length (feet) & (Line 1-2, Line 3-4) $=(2000 \mathrm{ft}, 2500 \mathrm{ft})$ \\
\hline Line impedance & See [7] for 4-wire configuration \\
\hline Grounding impedance & $\begin{array}{c}\text { (Substation, Bus 3, Bus 4)= } \\
(1 \mathrm{Ohm}, \text { Ungrounded, 25 Ohm })\end{array}$ \\
\hline $\begin{array}{c}\text { Phase-to-neutral } \\
\text { loads of bus 4 }\end{array}$ & $\begin{array}{c}(\mathrm{Pa}, \mathrm{Pb}, \mathrm{Pc})=(10 \mathrm{~kW}, 12 \mathrm{~kW}, 15 \mathrm{~kW}) \\
(\mathrm{Qa}, \mathrm{Qb}, \mathrm{Qc})=(7.5 \mathrm{kVar}, 9 \mathrm{kVar}, 11.25 \mathrm{kVar})\end{array}$ \\
\hline Taps & $\left(\mathrm{Tap}_{a}, \mathrm{Tap}_{b}, \mathrm{Tap}_{c}\right)=(-5,-11,-16)$ \\
\hline Transformer Power & $1000 \mathrm{kVa}$ \\
\hline Transformer Voltage & $20 \mathrm{kV} / 400 \mathrm{~V}$ \\
\hline Transformer impedance & $0.01+0.04 \mathrm{j}(\mathrm{pu})$ \\
\hline
\end{tabular}

Table 2

Voltage (V) calculated by Simulink and the proposed model.

\begin{tabular}{|lllllll|}
\hline Method & $\mid$ V2ab & $\mid$ V2bc & $\mid$ V2ca & $\mid$ V4an & $\mid$ V4bn & $\mid$ V4cn \\
\hline
\end{tabular}

\begin{tabular}{llllllll}
\hline Simulink & 19999.46 & 19999.47 & 19999.16 & 235.5856 & 228.9303 & 216.1647
\end{tabular}

$\begin{array}{llllllll}\text { Proposed } & 19999.47 & 19999.48 & 19999.17 & 235.5851 & 228.9307 & 216.1627\end{array}$ 


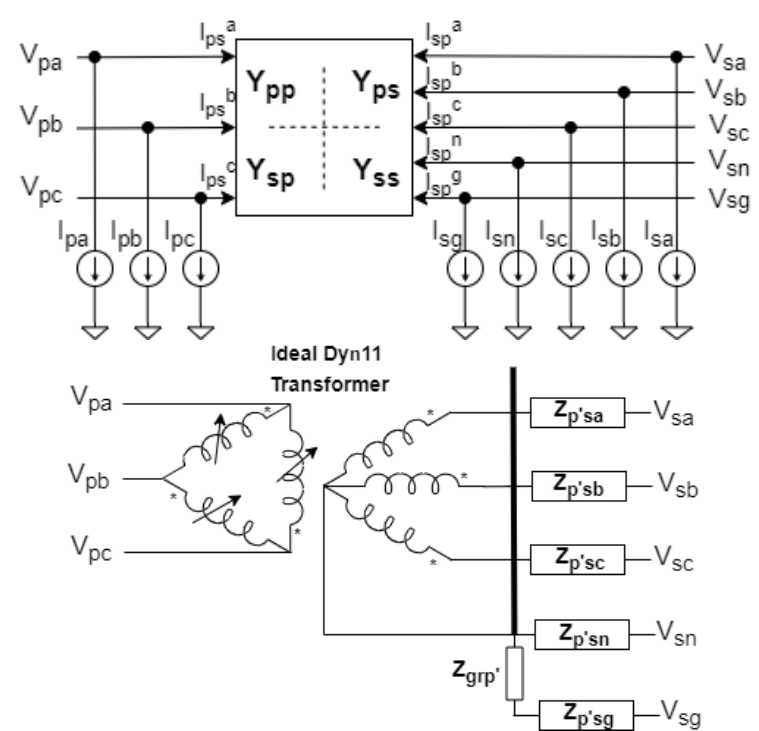

Fig. 2. From top to bottom: a) Equivalent circuit of the OLTC transformer, b) Part of the network of Fig. 1 represented by the equivalent circuit of OLTC transformer.

\section{Case study in a large network}

The proposed OLTC transformer model is tested in a large-scale network using the $Z_{\mathrm{BUS}}$ power flow method of section 4. The network consists of the IEEE 8500-Node MV network [7] and the IEEE European LV 906-Bus test feeder [7]. The MV and LV networks are connected through a 12.47/0.4kV Dyn11 OLTC transformer between the MV bus "L3312692" and the LV bus "SourceBus". Clarifications about the networks are provided below:

- In LV network, the grounding impedance of the transformer is $1 \mathrm{Ohm}$, while each load bus is grounded with $25 \mathrm{Ohm}$. The MV network is considered perfectly grounded at each bus.

- Data about the loads of MV network are provided in [7]. The loads of LV network correspond to the load profile given in [7] at 12.00 noon.

- Data about the lines of both networks are given in [7]. It is clarified that the MV bus "L3312692" is the most remote MV bus and it is single-phase. Thus, we modified it to a three-phase bus to enable the connection of the transformer.

- In IEEE 8500-Node network, the tap position of the step voltage regulators (SVRs) at the substation is $\{16,16,16\}$, while for the other three SVRs it is $\{7,7,7\}$.

- The OLTC transformer has the following characteristics: $\mathrm{S}=800 \mathrm{kVA}, \mathrm{Z}=0.004+0.04 \mathrm{j}$ (pu), $U / u=12.47 / 0.4 \mathrm{kV}$.

Table 3 shows the iterations required for the power flow to converge with an accuracy of $10^{-5} \mathrm{pu}$, for several tap sets $\left(\operatorname{Tap}_{a}, T a p_{b}, T_{a p}\right)$. In all cases a flat start was considered. It is confirmed that the proposed transformer model implemented in a $\mathrm{Z}_{\mathrm{BUs}}$-based power flow method presents fast convergence given the large size of the network and the ill-condition of the IEEE $8500 \mathrm{MV}$ network.

Figure 3 depicts the convergence process of the algorithm, when the taps of the three phases (they are denoted with red numbers in the figure) are readjusted every 16 iterations. At the beginning of the process (iteration 0) a flat start was considered. The figure confirms the fast convergence of the proposed OLTC transformer model.

With the proposed transformer model, the $\boldsymbol{Y}_{\boldsymbol{B} U \boldsymbol{S}}$ matrix is not refactorized or inversed after every tap change saving significant calculation time. Indicatively, the inversion of the $\boldsymbol{Y}_{\boldsymbol{B} U S}$ matrix that includes the investigated IEEE 8500node and IEEE 906-Bus networks is around 5 minutes in Matlab using a personal computer with the following specifications: 64-bit Intel Core i7, 3.4GHz CPU, 16 GB RAM. Therefore, several power flow applications that require sequential tap changes e.g voltage stability analysis, heuristic optimization, OPF, VVC, OFR [2], [3] speed up significantly with the proposed OLTC transformer model.

Table 3

Required iterations for several tap numbers

\begin{tabular}{|ccccccc|}
\hline Taps & $(0,0,0)$ & $(-15,-5,-5)$ & $(-14,-3,-2)$ & $(-16,-16,-16)$ & $(-8,3,4)$ & $(-4,7,8)$ \\
\hline Iterations & 15 & 16 & 16 & 16 & 15 & 15 \\
\hline
\end{tabular}

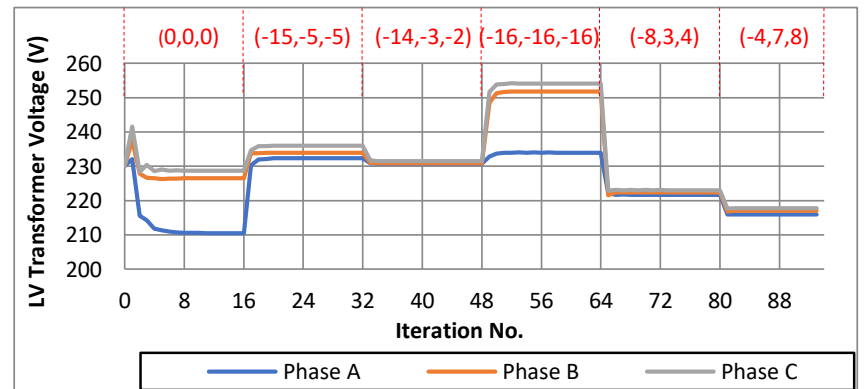

Fig. 3. Convergence process of the LV transformer voltage. The transformer connects the IEEE 8500-Nodes with the IEEE 906-Bus networks. The taps (denoted with red numbers) vary each 16 iterations.

\section{Conclusion}

This short communication presents an OLTC transformer model, which enables the realistic representation of the 3wire MV and 4-wire multigrounded LV networks into a single $Y_{\text {BUS }}$ matrix. Its advantage is that the tap changer is simulated outside the $Y_{\text {BUS }}$ matrix, thus avoiding the timeconsuming refactorization or inversion after every tap variation.

\section{References}

[1] M. Sojer, "Voltage Regulating Distribution Transformers as new Grid Asset", Procedia Engineering, Vol. 202, 2017, Pages 109-120, 2017.

[2] R. A. Jabr, I. Džafić and S. Karaki, "Tracking transformer tap position in real-time distribution network power flow applications", IEEE Transactions. on Smart Grid, vol. 9, no. 4, pp. 2442-2452, 2018.

[3] I. Huseinagić et al., "A compensation technique for unsymmetrical three-phase power flow", International Symposium on Industrial Electronics (INDEL), 2016.

[4] M.J.E. Alam et al, "A three-phase power flow approach for integrated 3-wire MV and 4-wire multigrounded LV networks with rooftop solar PV", IEEE Trans. Power Syst., vol. 28, no. 2, pp. 1728-1737, 2013.

[5] E.E Pompodakis, G.C Kryonidis, M. C Alexiadis, "A comprehensive load flow approach for grid-connected and islanded AC microgrids", Trans. Power Systems, vol. 35, no. 2, pp. 1143-1155, 2020.

[6] L.V. Bardoza et. al, "Load Tap Change Transformers: A Modeling Reminder", IEEE Power Engineering. Review, Vol. 21, Issue 2, 2001

[7] IEEE PES. (2020, May), "IEEE PES AMPS DSAS Test Feeder Working Group", [Online]. Available: https://site.ieee.org/pestestfeeders/resources/

[8] E. Pompodakis, G. Kryonidis, C. Demoulias, M. Alexiadis, "A Generic Power Flow Algorithm for Unbalanced Islanded Hybrid AC/DC Microgrids", IEEE Transactions on Power Systems (Early Access), DOI: 10.1109/TPWRS.2020.3012815, July 2020. 